\title{
A Note on Intradermal Injections
}

B. Moiser.

The use of short needles has been given a good trial here over several years, but has now been discarded on account of leakage, and also on account of the objections raised by the patients to the multiple punctures.

Instead, an ordinary No. 23 needle is used, the skin is pinched up into a mound, and the needle introduced once only, the point being pushed into various parts of the corium from the interior. The point of the needle can be felt by the pinching thumb and finger, and can be adjusted to a nicety. Leakage is reduced to a minimum, the preparation is distributed into the skin over quite a large area, and the patients do not object.

Care is necessary not to push the needle through the skin, and so puncture the operator's own finger or thumb. It is advisable to point the needle towards the space between finger and thumb. 